\title{
Examining Knowledge Transfer between Thermodynamics and Mathematics
}

Dr. Alexander John De Rosa, Stevens Institute of Technology (School of Engineering and Science)

Alexander De Rosa is a Teaching Assistant Professor in Mechanical Engineering at Stevens Institute of Technology. Alex specializes in teaching in the thermal-fluid sciences and has a background in experimental combustion. He gained his PhD in 2015 from The Pennsylvania State University in this area. 


\title{
Examining Knowledge Transfer between Thermodynamics and Mathematics (WIP)
}

\begin{abstract}
It is well-established that students have difficulty transferring knowledge and skills between courses in their undergraduate curriculum. At the same time, many college-level courses only concern material relating to the course itself and do not cover how this material might be used elsewhere. It is unsurprising, then, that students are unable to transfer and integrate knowledge from multiple areas into new problems as part of capstone design courses for example, or in their careers. More work is required to better enable students to transfer knowledge between their courses, learn skills and theory more deeply, and to form engineers who are better able to adapt to new situations and solve "systems-level" problems. In this investigation, students in a sophomore thermodynamics class were presented with a sequence of problems that required skills learned in calculus courses to solve. Without guidance, students were initially asked to solve a thermodynamics problem before being given a prompt to activate their prior knowledge and a second chance to solve the problem. Prompting students to activate their prior knowledge was partially successful in improving their performance on the in-class problems but did not significantly improve overall problem solving success. Based on post-activity survey data, a majority of students stated that they felt they had many of the skills needed to solve the thermodynamics problem but that a lack of learning applications in the math curriculum, as well as the large gap in time between completing their math courses and thermodynamics were barriers to their success.
\end{abstract}

\section{Introduction}

In many courses across engineering curricula, students are expected to be able to apply knowledge and understanding from prior courses to solve problems in the current course. In mechanical engineering for example, students are required to transfer mathematical skills from the first-year curriculum into later, discipline specific courses such as heat transfer or fluid mechanics in order to solve various forms of differential equations. A problem often arises however in that students are unable to solve these problems in which knowledge must be transferred, or that they fail to see the connections between these topics and those covered in prior classes [1,2].

At many institutions, the core courses offered in first-year engineering curricula, such as mathematics and physics, are taught by faculty from outside the major of the student. This can lead to a lack of discipline-specific applications being taught as well as a lack of discussion of how the topics at hand connect to the engineering curriculum at large. It should not then be surprising that the links between these foundational courses and later discipline-specific classes are not clear to the students. In such cases, students are unable to leverage their prior knowledge learned in these classes in new situations and some form of remediation is required.

Problems with knowledge transfer can be found across the engineering curriculum as well as in various other fields of study [3]. Of particular interest here however is the connections students make between their foundational mathematics classes and later engineering courses. A summary 
of student understanding of, and ability to transfer, knowledge relating to integration is given by Orton from the mathematics perspective [4]. The author notes student difficulties in using skills such as integration in new applications and suggests revisiting fundamental mathematical concepts and reinforcing them throughout a student's education. Other investigators $[5,6]$ observed that while students might possess the requisite mathematical knowledge, the problems they have in applying this knowledge are likely related to students' inability to see how this work properly applies in a given context, including which assumptions and approximations they are required to make.

In the context of foundational physics education, various authors have examined student understanding of mathematical concepts and how they relate to higher level physics and thermalphysics classes. Christensen and Thompson [7] asked students questions about integration in both a math $(x, y)$ and thermodynamics $(P, V)$ framework. They found that students often lacked the prerequisite mathematical knowledge or the ability to apply it correctly. This lack of prior knowledge hampered student ability to solve the related physics problems. Several other studies from the physics community have also reported students having difficulties with applying mathematical knowledge to thermodynamics principles such as work done, and other state and process functions [8,9]. An important recommendation from this work is that the authors suggest the use of "resource activation" (prior knowledge activation) as a potentially helpful tool in remedying these issues. The authors did not explore this idea further however and it does not seem to have been followed up by other investigators.

A related body of work does exist in the engineering field concerning knowledge transfer and deeper conceptual learning [10,11]. There is still significant work to be done however in developing tools to help students develop a deeper understanding of concepts, and to improve their ability to transfer knowledge between courses. In this study we aim to develop these tools that enable knowledge transfer and activate the prior knowledge of students. In particular, the ability of students to transfer knowledge from mathematics into thermodynamics, and to solve problems of integration is examined in order to pilot these newly developed activities.

\section{Methodology}

This study was conducted within a sophomore-level thermodynamics class. A total of 177 students made up the sample population. These students were enrolled in a variety of majors, including mechanical, civil, biomedical, software, industrial, computer and software engineering. In order to both assess then promote knowledge transfer, an activity was designed in which students were required to solve a thermodynamics problem requiring transfer of mathematical concepts before having their prior knowledge activated with a relevant prompt. The students then attempted to solve the initial problem with the hypothesis that provision of the prompt would improve their ability to transfer the required mathematical skills and solve the problem. The students were not given guidance from the instructor during the activity and worked individually through the problems. As an incentive to complete this activity and to allow creativity, students were awarded credit for completing the assignment independent of their actual score on the three problems involved. 
The force due to the pressure exerted by a gas on the walls of a container is $F=P^{*} A$, where $P$ is the pressure in Newtons $/ \mathrm{m}^{2}$ and $A$ is the area in $\mathrm{m}^{2}$.

A frictionless piston containing an ideal gas, which follows the equation of state $P V=m R T$, is slowly compressed under isothermal conditions so that the gas is always in equilibrium with the surroundings.

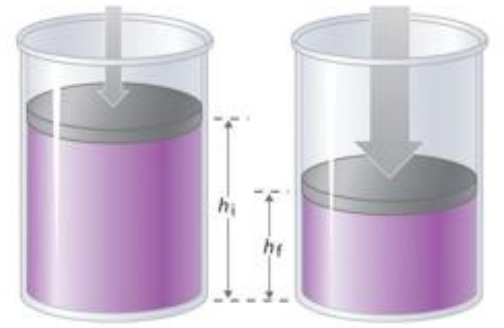

Derive an expression for the amount of work needed to compress the piston a distance of $\Delta \mathrm{h}$.

Figure 1: Thermodynamics (boundary work) problem statement

The thermodynamics problem chosen as the basis of this study was that of "boundary work" as solutions to this problem typically require students to substitute and integrate in order to solve them. These are the skills (integration and substitution) that must be transferred by the students. Part-way through the course but before covering the boundary work topic formally in class, students were presented with an in-class activity in three parts. In the first step, students were asked to solve the problem of isothermal boundary work as shown in Figure 1. The solution to this problem is detailed in equations 1-3. By this point in the course, ideas such as isothermal processes and thermal equilibrium had been discussed along with the ideal gas law.

$$
\begin{gathered}
W=\int_{V_{1}}^{V_{2}} P d V \\
P V=m R T=k(\text { constant under } \text { isothermal conditions }) \\
W=\int_{V_{1}}^{V_{2}} \frac{k}{V} d V=k \cdot \ln \frac{V_{2}}{V_{1}}
\end{gathered}
$$

After attempting to solve this initial problem without guidance, the second step was to prompt the class with the relevant prior knowledge needed to solve for the boundary work. A related problem that students had solved in the mathematics curriculum was chosen that demonstrated the need to both substitute and integrate. This problem was provided by the faculty teaching integral calculus as follows: The force due to friction $F(N)$ needed to move an object with mass $m(\mathrm{~kg})$ across a surface is $F=\mu \mathrm{m}$, where $\mu(\mathrm{N} / \mathrm{kg})$ is some coefficient that depends upon surface properties and the gravitational constant. Consider a block of wax being pushed across a surface, leaving behind a trail of wax as it travels. Its mass is related to the distance the wax has been pushed $(x)$ and is given by $m(x)=50-0.5 x$. Assuming that the coefficient $\mu=2$, how much work is needed to push the block 100m across this surface? This problem and its solution are further detailed in equations $4 \& 5$. Again, students were asked to solve this problem without guidance.

$$
\begin{gathered}
W=\int_{0}^{100} F(x) d x \text {, where } F(x)=\mu \cdot m(x) \text { and } m(x)=50-0.5 x \\
W=\int_{0}^{100} 2 \cdot(50-0.5 x) d x=5000 \mathrm{~J}
\end{gathered}
$$


In the final step of the activity, students were asked to solve the initial boundary work problem again in order to assess the impact of the mathematics prompt on their problem-solving success. At the end of the activity, the solutions to the problems were formally presented to the students and a survey distributed asking student the following questions:

1. Did solving the math problem help you to solve the thermodynamics problem? If yes, please explain in what way. If no, why not?

2. Do you agree or disagree that you had sufficient knowledge/skills to solve the problem without instructor guidance? Why or why not?

3. When did you study integral calculus?

4. Approximately how many times have you used these skills (integration \& substitution) between this class and when you took your calculus class?

Student ability to use mathematical concepts and the efficacy of the prior knowledge activation activity was assessed by grading each element of the in-class assignment on a scale of 0-4.

Students were given a point each for recognizing the need to apply the equation "work $=$ force $\mathrm{x}$ distance", the need to integrate to solve the problem, and realizing the need to substitute in for the pressure in terms of volume. The final point was awarded for a correct overall solution.

\section{Results}

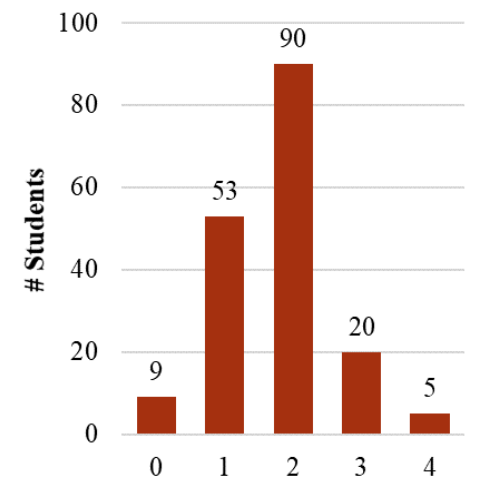

(a) Attempt 1

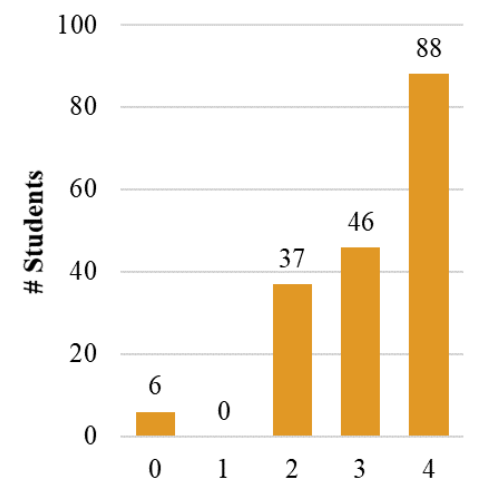

(b) Math problem

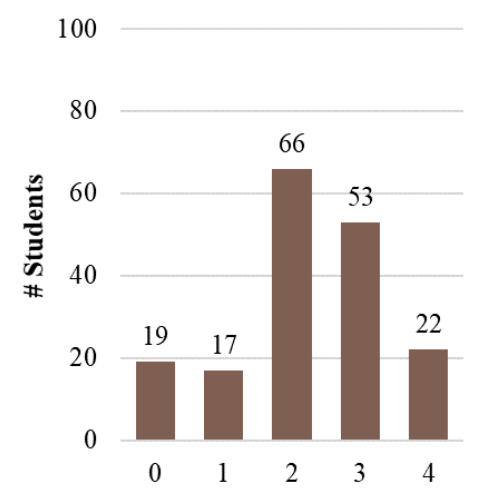

(c) Attempt 2

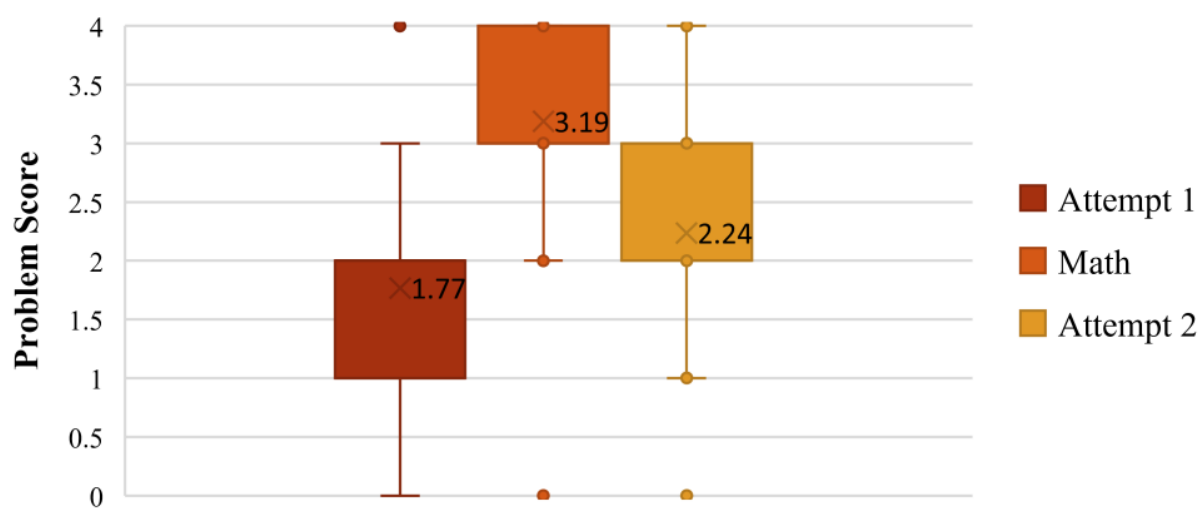

(d) Popoulation scores in each phase of the activity, mean score indicated and outliers shown.

Figure 2: Student scores (out of four points) in the three phases of the in-class activity (n=177). Histograms shown (a-c) and (d) box and whisker plot. 
Figure 2 details the scores of students on the in-class activity. Four points was the maximum possible point score. Only five students achieved a full score (entirely correct solution) on the first attempt at the boundary work problem (Fig.2a). Student responses to the mathematics problem were of higher quality and 88 of 177 students, $50 \%$ of the class, solved this problem correctly when it was framed in a mathematical context (Fig.2b). Seventy-six percent of the class scored three or more points on the math problem. These high scores indicate that the majority of students do possess the requisite mathematical skills necessary to solve the boundary work problem. Importantly, at the second attempt at solving the boundary work problem, the distribution of student scores is shifted to higher totals with 75 students scoring three or more points at the second attempt compared to only 25 students scoring three or more on the first attempt at solving the problem (Fig.2c). This shift is also seen in Fig.2d where the mean score on the thermodynamics problem increases from 1.77 to 2.24 points and the general distribution of scores is now centered on an increased median score. This result is indicative of the fact that providing the math problem as a prompt was successful in activating student prior knowledge and aided the class in solving the thermodynamics problem.

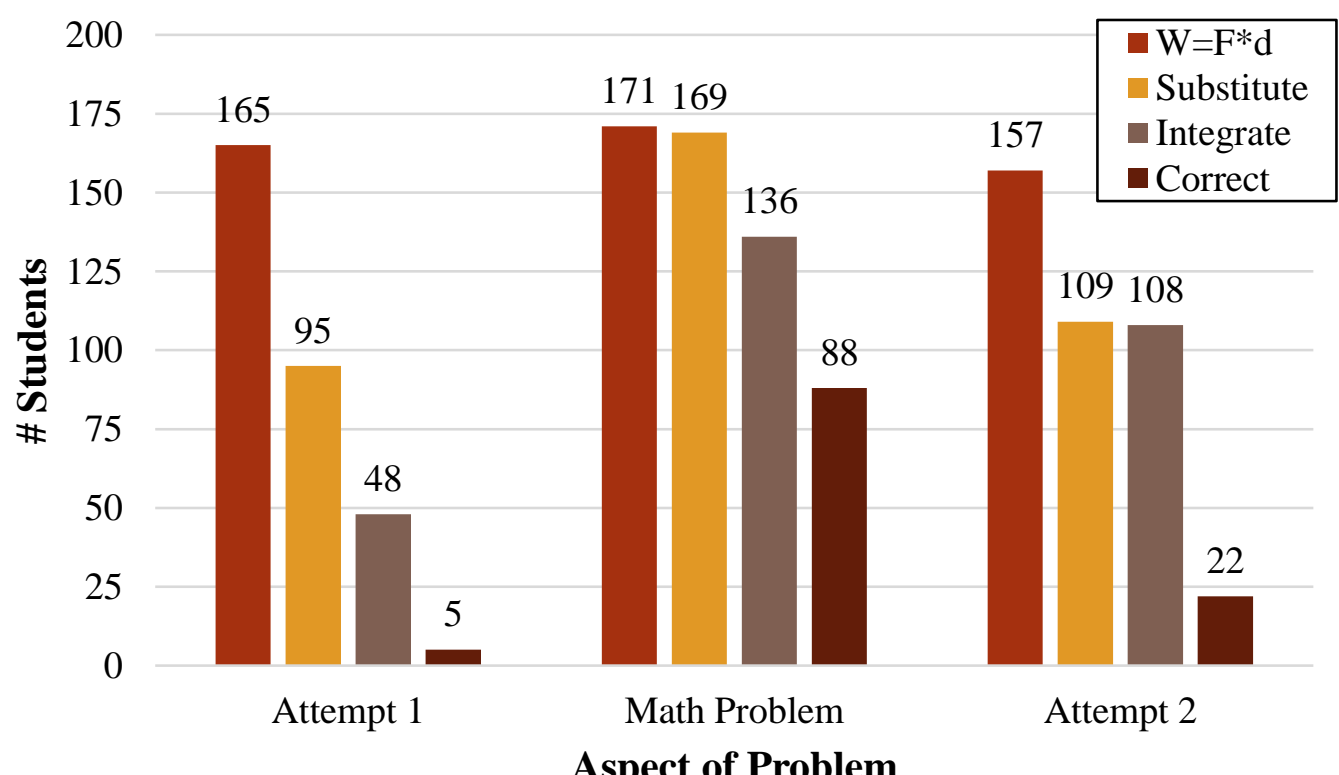

Figure 3: Student performance on the 3 parts of the in-class activity as measured by their ability to recognize $W=F^{*} d$, the need to substitute and integrate to solve the problem, and, finally the correctness of their solution $(n=177)$.

The performance of the students within the three phases of the activity is further detailed in Figure 3. In this plot, student ability to recognize individual concepts within each phase of the problem is shown - in each phase, the number of students who recognized; "work = force $\mathrm{x}$ distance", the need to substitute, and integrate to solve the problem is detailed along with the number of students who also scored a point for a correct solution. Again the improvement in student solutions through the in-class activity is clearly observed as the number of conceptual elements recognized increases between attempt 1 and 2 after prior knowledge activation and knowledge transfer via the math problem. Of the five students who solved the problem correctly 
at the first attempt, all five again solved the problem correctly the second time i.e. the provision of the math problem did cause them to change their response or second-guess their solution.

In particular, the math problem was useful in showing students the need to integrate to solve the thermodynamics problem. The number of students who employed integration rose from 48 students in the initial attempt, to 108 in the second attempt at solving the boundary work problem. This result indicates that students perhaps did not initially realize the need to use integration in this application, and that the math problem prompted them to do so. While the math prompt was useful in this regard, and student performance on the second attempt improved, provision of the prompt did not dramatically increase the success of the students in solving the overall problem correctly.

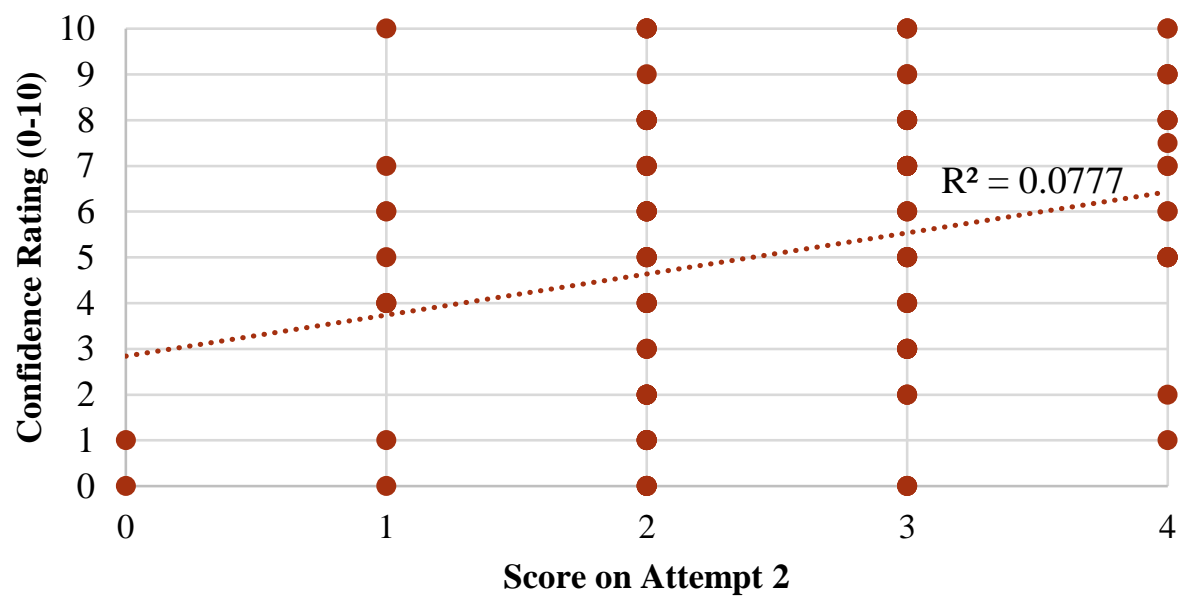

Figure 4: Student confidence in their solution (0-10) against their overall score on the second attempt at solving the thermodynamics problem.

After completion of the activity, students were asked to rate their confidence in their solution to the boundary work problem. As can be seen in Fig.4, student confidence in their problem solving ability had no observable correlation with their actual performance on the activity $\left(\mathrm{R}^{2}=0.0777\right)$. This result is important as it indicates the lack of understanding students have of these problems in that they do not know whether their solutions are correct or even if they are applying the correct method to solve the problem.

In addition to collecting numerical data regarding student performance on the sequence of inclass activities, survey data was also collected from the students regarding their perception of the problems and the skills involved in solving them. Figure 5 details student responses to two of these questions, namely whether students believed the math problem helped them in solving the thermodynamics problem and, secondly, whether the sample population believed they had the knowledge and skills required to solve the problem of boundary work. The results (Fig.5) indicate that the students overwhelmingly believed that they had the required knowledge to solve the problem and that the math prompt was helpful in enabling them to solve for the boundary work. In each case approximately $85 \%$ of the sample population ( 140/167 students) responded positively to each question. The survey responses did not include all students who completed the 
in-class activities as several students did not complete the surveys. This lead the "survey" sample to be slightly smaller than the "activity" sample (167 compared to 177 students).

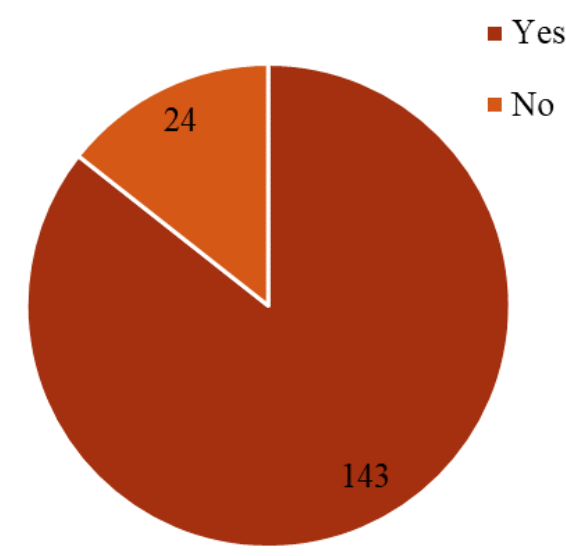

(a) Did solving the math problem help you to solve the thermodynamics problem?

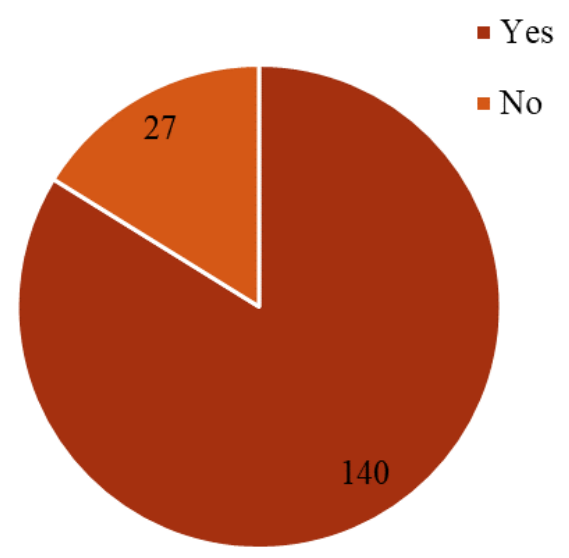

(b) Do you agree or disagree that you had sufficient knowledge/skills to solve the problem without instructor guidance?

Figure 5: Student responses to student survey questions ( $n=167)$.

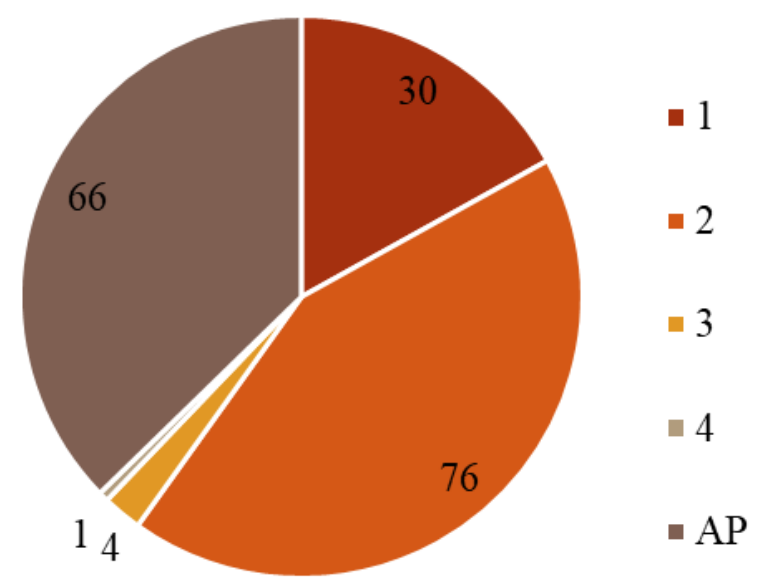

Figure 6: Student reporting of number of years between taking integral calculus and thermodynamics in years, or listed as AP credit $(n=177)$.

Given that students thought they had the skills required to solve these problems, it was interesting that the majority of them could not so successfully. It might be expected that the time between students taking thermodynamics and integral calculus, as well as the number of times they used integration skills in the intervening period would play a role in their ability to complete this activity. As such, these factors were assessed. Figure 6 shows the time, in years, between students taking their integral calculus class and enrolling in thermodynamics. For the majority of students (76/177) this period was two years given the placement of these courses within the typical course of study. A significant population however had never taken a calculus course at 
Stevens due to the acceptance of AP credit (66/177 students). For these students the gap between these courses is likely to be between two and three years and could lead to problems in transferring knowledge successfully as they may have forgotten this material in the intervening time period or not been taught relevant applications to engineering in their AP courses. Having said this however, 4/5 students who solved the problem correctly at the first attempt had used AP credit instead of taking the integral calculus course at Stevens.

While the time lapse between learning concepts of integration and needing to use them to solve thermodynamics problems is potentially significant, students did report using these skills many times in the intervening period. Almost all students responded that they had used integration as a skill many times since taking their fundamental mathematics courses. A significant problem with this however was that almost $50 \%$ of students responded that they had only used these skills in the math sequence and not as often in major-specific courses. Responses such as that given here were typical of comments made by students: "I have used integration skills a few times in my Differential Equations [course] and [in] Probability/Statistics a few times. However, outside of my math classes, I rarely have to use my integration and substitution skills." Evidently this represents a potential problem in that while students are learning the skills necessary to complete this activity, they might not be learning specific applications of how to use these skills in contexts outside of mathematics. Future work will investigate the degree to which applications are taught in the math curriculum as well as whether students from different majors experience these skills differently (the sample population detailed here is from a thermodynamics class with a variety of student majors enrolled).

\section{Conclusions}

In order to promote knowledge transfer and to reinforce fundamental concepts in engineering, a sequence of in-class activities was designed in which students attempted to solve a thermodynamics problem, that of boundary work, without guidance before having their prior knowledge activated with a relevant prompt. Students were then given a second opportunity to solve the thermodynamics problem after having their prior knowledge activated in order to assess the efficacy of this technique in promoting knowledge transfer. In this case, the specific problem students attempted to solve was that of isothermal boundary work, a problem which requires both substitution and integration to solve. Students were prompted to recall their prior knowledge using a related mathematics problem that also required use of these skills.

Students $(n=177)$ were not initially able to solve the thermodynamics problem without guidance (5/177 students answered correctly). Eighty-eight students were however able to solve the mathematics problem and provision of this prompt lead to 22/177 students solving the thermodynamics problem successfully at the second attempt. While the sequence of in-class activities did not dramatically improve the ability of students to solve the boundary work problem, it did remind many students of the skills they needed to solve the problem and 75 students scored 3 or more points (out of a possible 4) on their second attempt at the problem as opposed to only 25 students scoring 3 or more on the first attempt. In this manner, provision of the math prompt to aid in solving the thermodynamics problem by promoting knowledge transfer was partially successful. 
Student survey data indicated that the sample population overwhelmingly believed ( $85 \%$ of respondents) that they had the required skills to solve this problem. Students also reacted positively to the use of the math problem to enable their prior knowledge. Again, $85 \%$ of survey respondents suggested that the math prompt was useful in reminding them of the skills necessary to solve for the boundary work. Given this result, it was interesting that only 22/177 students solved the boundary work problem correctly. Factors such as the length of time between taking mathematics courses and thermodynamics, as well how often students had used their integration skills in the intervening period were considered. Results indicated that while students do use integral calculus extensively in their mathematics courses, they are rarely called upon to apply these concepts in their core engineering classes, leading to potential lack of contextual understanding of integration and how it applies in a given situation.

\section{Future Work}

Future work in this study will expand this dataset to include a larger survey population and by developing new tools and exercises to promote knowledge transfer. Student understanding of the various skills involved will also be assessed pre- and post-class using targeted concept inventories. Work will also be completed in order to correlate student performance in thermodynamics and their ability to transfer knowledge with other indicators such as GPA, grades in the math sequence, and by examining differences in students as grouped by major, gender and URM status.

\section{Acknowledgements}

This work was conducted under IRB 2019-018(N) and grew out of work started under the NSF Foundations Project, Award \#1524656, at Stevens Institute of Technology and initiated with the help of Dr. S. Lee and Dr. D. Serbin.

\section{Bibliography}

1. Bransford, John D., and Daniel L. Schwartz. "Chapter 3: Rethinking transfer: A simple proposal with multiple implications." Review of research in education 24.1 (1999): 61-100.

2. Detterman, D. K., \& Sternberg, R. J. (1993). Transfer on trial: Intelligence, cognition, and instruction. Ablex Publishing.

3. D. N. Perkins \& G. Salomon 1988 Teaching for transfer. Educational Leadership 46 (1): 2232.

4. Orton, A. (1983). Students' understanding of integration. Educational Studies in Mathematics, 14(1), 1-18.

5. Rebello, N. S., Cui, L., Bennett, A. G., Zollman, D. A., \& Ozimek, D. J. (2007). Transfer of learning in problem solving in the context of mathematics and physics. Learning to Solve Complex Scientific Problems, 223-246.

6. Schoenfeld, A. (1985). Mathematical Problem Solving. New York: Academic Press.

7. Christensen, W. M., \& Thompson, J. R. (2010). Investigating student understanding of physics concepts and the underlying calculus concepts in thermodynamics. In Proceedings of the 13th Annual Conference on Research in Undergraduate Mathematics Education. 
8. Loverude, M. E., Kautz, C. H., \& Heron, P. R. (2002). Student understanding of the first law of thermodynamics: Relating work to the adiabatic compression of an ideal gas. American Journal of Physics, 70(2), 137-148.

9. Meltzer, D. E. (2004). Investigation of students' reasoning regarding heat, work, and the first law of thermodynamics in an introductory calculus-based general physics course. American Journal of Physics, 72(11), 1432-1446.

10. Ellis, G. W., Rudnitsky, A., \& Silverstein, B. (2004). Using concept maps to enhance understanding in engineering education. International Journal of Engineering Education, 20(6), 1012-1021.

11. Turner, W., \& Ellis, G. (2003), Helping Students Organize And Retrieve Their Understanding Of Dynamics, In Proceedings of the 2003 ASEE Annual Conference, Nashville, Tennessee. 